\title{
Computational analysis of the functional role of cerebellar Basket-type inhibitory connections: compensation or coding?
}

\author{
Rachael Wilcox ${ }^{1,2^{*}}$, Hugo Cornelis ${ }^{2}$, James M Bower ${ }^{1,2}$ \\ From Nineteenth Annual Computational Neuroscience Meeting: CNS*2010 \\ San Antonio, TX, USA. 24-30 July 2010
}

Despite the morphological changes observed within the cerebellum over the course of evolution, its circuitry with respect to its neuronal types, have remained a remarkable constant [1]. In particular, the relationship between the geometry of the Purkinje cells and the parallel fiber projection of the cerebellar granule cells has been observed in all vertebrates [2]. However, one clear comparative difference is the fact that basket-type inhibitory connections to the soma of the Cerebellar Purkinje cells are only found in birds and mammals [3]. The objective of this study was to test the model-based predication [4] that these basket inhibitory inputs serve to control the excitatory effects of the larger number of parallel fiber inputs found in birds and mammals.

In the first phase of this project, cell-counting techniques have been used to obtain the ratio of granule cells to Purkinje cells in a wide range of vertebrate animals. These results suggest that, in fact, larger GC:PC ratios are found in birds and mammals. The results to be presented here were obtained by first using 3D neuronal reconstruction techniques to characterize the shapes of the dendrites and somata of cerebellar Purkinje cells from fish, turtles, rats and birds. These morphologies were then used to create compartmental single cell models which, in turn, were used to explore the cells passive electrical properties. Parameters explored include the maximal amplitude, time to reach maximal amplitude, voltage drops, and the standard deviation from the average membrane potential during spiking. All procedures were conducted under the approval and supervision of the Animal Welfare and Use Committee. All simulations were conducted using GENESIS 3.0. The results suggest that the Purkinje cells of birds and mammals are more electronically compact than are those of turtles and fish.

Taken together, these results support the hypothesis that the basket-type inhibitory connection evolved to compensate for both an increase in the number of parallel fiber inputs per Purkinje cell as well as in the computational speed of processing of the Purkinje cell. The results will be interpreted in the context of our network model of cerebellar cortical circuitry.

\section{Acknowledgements}

Supported by a grant from the National Science Foundation

\section{Author details}

${ }^{1}$ Neurosciences Institute, University of Texas at San Antonio, San Antonio, TX 78249, USA. ${ }^{2}$ Research Imaging Institute, University of Texas Health Sciences Center at San Antonio, San Antonio, TX 78229, USA

Published: 20 July 2010

\section{References}

1. Llinás RR, Walton KD, Lang EJ: Cerebellum. The Synaptic Organization of the Brain Shepard GM, editor. Oxford University Press., 5th 2004, 271-309.

2. Bell C: Evolution of cerebellum-like structures. Brain Behav Evol 2002, 59:312-326.

3. Larsell O: The Comparative Anatomy and Physiology of the Cerebellum. University of Minnesota Press. 1972

4. Santamaria F, Tripp P, Bower JM: Feedforward inhibition controls the spread of granule cell-induced Purkinje cell activity in the cerebellar cortex. J. Physiology 2007, 97:248-263.

doi:10.1186/1471-2202-11-S1-P141

Cite this article as: Wilcox et al:: Computational analysis of the functional role of cerebellar Basket-type inhibitory connections: compensation or coding?. BMC Neuroscience 2010 11(Suppl 1):P141. 